\title{
Biologic Entity Identifier Effective Date Range
}

National Cancer Institute

\section{Source}

National Cancer Institute. Biologic Entity Identifier Effective Date Range. NCI Thesaurus.

Code C93743.

The date and time span for when the biologic entity identifier is active. 